\title{
Vegetation Database of Temperate Deciduous Forests of the Göttinger Wald
}

\author{
Wolfgang Schmidt, Michaela Dölle \& Andreas Parth
}

\begin{abstract}
The Vegetation Database of Temperate Deciduous Forests of the Göttinger Wald (GIVD ID EU-DE-018) offers forest vegetation relevés obtained from various research projects between 1955-2011 in the Göttinger Wald (S-Lower Saxony, Germany). Single surveys as well as repeated surveys are included. Beech forests ecosystems on limestone with variable fractions of other broadleaved trees (e.g. Acer pseudoplatanus, Fraxinus excelsior) are characteristic. The database comprises classical phytosociological data, experimental ecological data and additional data for population-biological analysis.
\end{abstract}

Keywords: beech forest; Germany; limestone.

GIVD Database ID: EU-DE-018

Last update: 2012-05-09

\section{Vegetation Database of Temperate Deciduous Forests of the Göttinger Wald}

Scope: data obtained from various research projects during six decades including single surveys as well as repeated surveys

Status: completed and continuing

Period: 1955-2011

Database manager(s): Wolfgang Schmidt (wschmid1@gwdg.de); Michaela Dölle (mdoelle@gwdg.de); Andreas Parth (aparth@gwdg.de)

Owner: University of Göttingen

Web address: http://www.uni-goettingen.de/de/67090.html

Availability: free upon request

Online upload: no

Online search: no

Database format(s): MS Access

Export format(s): [NA]

Publication: Schmidt, W., 2009. Vegetation. In: Brumme, R., Khanna, P.K. (eds.) Functioning and Management of European Beech Ecosystems. Results from site specific long-term studies. Ecological Studies 208: 65-86.

Plot type(s): normal plots; time series

Plot-size range: $1-400 \mathrm{~m}^{2}$

Non-overlapping plots: 350

Estimate of existing plots: [NA]

Completeness: [NA]

Total plot observations: 700 Number of sources: 1

Valid taxa: 500

Countries: DE: $100.0 \%$

Guilds: all vascular plants: $100 \%$; bryophytes (terricolous or aquatic): $50 \%$

Environmental data: altitude: $100 \%$; slope aspect: $100 \%$; slope inclination: $100 \%$; microrelief: $20 \%$; soil depth: $20 \%$; surface cover other than plants (open soil, litter, bare rock etc.): $20 \%$; soil pH: $30 \%$; other soil attributes: $100 \%$

Performance measure(s): cover: $100 \%$; number of individuals: $10 \%$; biomass: $10 \%$

Geographic localisation: GPS coordinates (precision $25 \mathrm{~m}$ or less): $80 \%$; point coordinates less precise than GPS, up to $1 \mathrm{~km}: 20 \%$

Sampling periods: 1950-1959: 20.0\%; 1960-1969: 10.0\%; 1980-1989: 20.0\%; 1990-1999: 25.0\%; 2000-2009: 20.0\%; 2010-2019: 5.0\%

Information as of 2012-07-12; further details and future updates available from http://www.givd.info/ID/EU-DE-018

Wolfgang Schmidt (wschmid1@gwdg.de), Michaela Dölle* (mdoelle@gwdg.de), Andreas Parth (aparth@gwdg.de)

Silviculture and Forest Ecology of the Temperate Zones, University of Göttingen, Büsgenweg 1, 37077 Göttingen, GERMANY

*Corresponding author 